\title{
Meteosat Third Generation: presentation of the video detection chains
}

Hubert Gardette, Nadège Remoue, Antonio Accettura, Mark Wilson

Hubert J. Gardette, Nadège Remoue, Antonio Accettura, Mark Wilson, "Meteosat Third Generation: presentation of the video detection chains," Proc. SPIE 11852, International Conference on Space Optics - ICSO 2020, 118520E (11 June 2021); doi: 10.1117/12.2599157

SPIE Event: International Conference on Space Optics - ICSO 2021, 2021, Online Only 


\section{International Conference on Space Optics-ICSO 2020}

Virtual Conference

30 March-2 April 2021

Edited by Bruno Cugny, Zoran Sodnik, and Nikos Karafolas


\section{Meteosat Third Generation: presentation of the video detection chains}

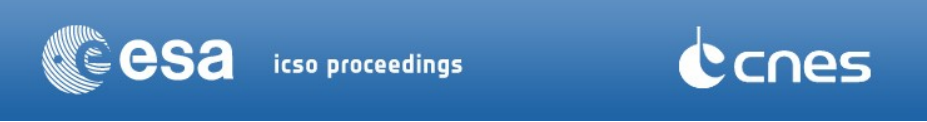




\title{
Meteosat Third Generation : presentation of the video detection chains
}

\author{
Hubert Gardette* ${ }^{\mathrm{a}}$, Nadège REMOUE ${ }^{\mathrm{a}}$, Antonio ACCETTURA ${ }^{\mathrm{b}}$, Mark WILSON $^{\mathrm{c}}$ \\ ${ }^{a}$ Thales Alenia Space, 5 allée des Gabians, BP 99, 06156 Cannes, France \\ ${ }^{\mathrm{b}}$ OHB, Manfred-Fuchs-Strasse 1, 82234 WESSLING/OBERPFAFFENHOFEN, GERMANY \\ c ESA/ESTEC, Keplerlaan 1,P.O. Box 299, 2200 AG NOORDWIJK, THE NETHERLANDS \\ *hubert.gardette@thalesaleniaspace.com, +33492923097
}

\begin{abstract}
Meteosat Third Generation (MTG) is the next challenging mission for accurate observation of the Earth dedicated to meteorology forecast.

MTG encompasses two different missions: MTG-I (Imaging) and MTG-S (Sounding). MTG-I optical payload counts the FCI (Flexible Combined Imager), a multi-spectral imager in the continuity of Meteosat Second Generation (MSG) with improved performance and additional spectral bands. MTG-S transports the IRS instrument which is an Infrared Fourier Transform Spectrometer dedicated to the sounding of the atmosphere inspired by the previous IASI mission.

Located in a geostationary orbit, the satellites are covering the full hemisphere for imaging (FCI) in less than 10 minutes in 16 different spectral bands from Visible to Very Long Wave IR at a resolution of $0.5-2 \mathrm{~km}$ and make a complete IR sounding of the atmosphere (IRS) in less than $1 \mathrm{~h}$ at a spatial resolution of $4 \mathrm{~km}$ and a spectral resolution of $0.625 \mathrm{~cm}-1$.

In this article, we describe the detection chains of FCI and IRS instruments which provide accurate sight to the remote sensing capabilities and thus aiming at achieving the objectives of MTG-I and S missions. Some particularities of designs and key performance are presented. MTG on ground tests have provided interesting results which are therefore presented. These results have been obtained after 10 years of intensive research and development at Thales Alenia Space (Prime FCI) \& OHB(Prime IRS), with the support and supervision of ESA and EUMETSAT.
\end{abstract}

This research and development work has been performed under an ESA contract.

Keywords: Meteosat, MTG, FCI, IRS, detector, visible range, Near Infrared Range, Infrared, video chain, Infrared Sounding, Earth Atmosphere, Earth Observations

\section{INTRODUCTION}

Meteosat Third Generation (MTG) is the next challenging mission for accurate observation of the Earth dedicated to meteorology Nowcasting and Forecasting.

MTG encompasses two different missions: MTG-I (Imaging) ${ }^{[1][2]}$ and MTG-S (Sounding). MTG-I optical payload counts a Flexible Combined Imager (FCI) and a Lightning Imager (LI). The FCI is a multi-spectral imager in the continuity of Meteosat Second Generation (MSG) with improved performance and additional spectral bands. The FCI is developed, integrated and tested by Thales Alenia Space in France, whereas the LI is developed by Leonardo (Italy). MTG-S carries the IRS instrument which is an Infrared Fourier Transform Spectrometer dedicated to the sounding of the atmosphere inspired by the previous IASI mission ${ }^{[3][4]}$. The IRS instrument is being developed, integrated and tested by OHB. encompasses two major sub-assemblies provided by Thales Alenia Space in France: the Interferometer Assembly and the Detection Electronic Assembly.

MTG satellites are located on a geostationary orbit. MTG-I satellite images the full hemisphere with FCI in less than 10 minutes in 16 different spectral bands from Visible to Very Long Wave IR at a resolution of $0.5-2 \mathrm{~km}$, while MTG-S satellite makes a complete IR sounding of the atmosphere (IRS) in less than $1 \mathrm{~h}$ at a spatial resolution of $4 \mathrm{~km}$ and a spectral sampling of $0.625 \mathrm{~cm}-1$. 
In this article, we focus on the detection chains of FCI and IRS instruments which provide accurate sight to the remote sensing capabilities and thus aiming at achieving the objectives of MTG-I and MTG-S missions ${ }^{[5][6]}$ for meteorological applications. This represents a total of 5 detection chains for FCI and 2 detection chains for IRS, all being under Thales Alenia Space responsibility for MTG. The paper presents at the same time the different particularities, design hardware and performance of FCI and IRS detection chains, as it is an interesting way to compare the two instruments and the two missions, even though the need and the concepts are specific to each instrument.

The spectral bands of interest of each mission, and how it is dispatch into multiple detection chains, are presented in a first paragraph. Then, the specific design and hardware of each focal plane array is described, in particular the pixels design and the different detectors disposition in the field of view of the instrument. Then the global video chains architectures are introduced to present how the photo-electronic information is processed from the analog acquisition to the digitalization.

After these descriptions, paragraph four will indicate some significant remote functionalities implemented in the video chains (at detector or electronic processing level), as well as some interesting parameters that can be adjusted by telecommand in flight in complement to the ones frozen on ground.

Some recent MTG PFM on ground tests, achieved in Thales Alenia Space premises, have provided interesting results which are therefore presented in the last paragraph. These results have been obtained after 10 years of intensive research and development at Thales Alenia Space (Prime FCI) \& OHB (Prime IRS), with the support and supervision of ESA and EUMETSAT.

\section{SPECTRAL BANDS OF INTEREST OF FCI AND IRS}

The MTG mission is a wide band mission allowing addressing various aspects of the meteorology on Earth. For imaging, the FCI instrument counts 16 narrow spectral bands from $0.4 \mu \mathrm{m}$ to $13.3 \mu \mathrm{m}$. All 16 bands are acquired simultaneously. For sounding, the IRS instrument counts 2 wide spectral bands in the IR region. Those spectral bands are indicated in Table 1. We have designed a retina (= photovoltaic material with its readout circuit) for each band of interest.

On FCI, retinas presenting close spectral bands have been integrated in a same packaging to constitute a Detector Assembly $(\mathrm{DA}=$ retina + optical filter + mechanical support + electrical connector $)$. The DA packaging is then constituted of the retina, the optical bandpass filter, the mechanical support and the electrical connector with flexible cable between the connector and the retina. On FCI, five DA are necessary to cover the need.

On IRS, it is more classical as the two large spectral bands imposed to have two DAs with only one retina per DA. One DA dedicated for MWIR range and one DA dedicated for LWIR range. Hence, two DA are necessary on IRS. 


\begin{tabular}{|c|c|c|c|c|c|}
\hline Instrument & DA & Channel & $\begin{array}{l}\text { Wavelength min } \\
{[\mu \mathrm{m}]}\end{array}$ & $\begin{array}{l}\text { Wavelength max } \\
{[\mu \mathrm{m}]}\end{array}$ & $\begin{array}{c}\text { spectral bandwidth } \\
{[\mu \mathrm{m}]}\end{array}$ \\
\hline \multirow{16}{*}{$\mathrm{FCl}$} & \multirow{5}{*}{ VIS } & VIS0.4 & 0,414 & 0,474 & 0,06 \\
\hline & & VIS0.5 & 0,49 & 0,53 & 0,04 \\
\hline & & VIS0.6 & 0,615 & 0,665 & 0,05 \\
\hline & & VIS0.8 & 0,84 & 0,89 & 0,05 \\
\hline & & VIS0.9 & 0,904 & 0,924 & 0,02 \\
\hline & \multirow{3}{*}{ NIR } & NIR1.3 & 1,365 & 1,395 & 0,03 \\
\hline & & NIR1.6 & 1,585 & 1,635 & 0,05 \\
\hline & & NIR2.2 & 2,225 & 2,275 & 0,05 \\
\hline & \multirow{3}{*}{ IR1 } & IR3.8 & 3,6 & 4 & 0,4 \\
\hline & & IR6.3 & 5,8 & 6,8 & 1 \\
\hline & & IR7.3 & 7,1 & 7,6 & 0,5 \\
\hline & \multirow{2}{*}{ IR2 } & IR8.7 & 8,5 & 8,9 & 0,4 \\
\hline & & IR9.7 & 9,51 & 9,81 & 0,3 \\
\hline & \multirow{3}{*}{ IR3 } & IR10.5 & 10,15 & 10,85 & 0,7 \\
\hline & & IR12.3 & 11,95 & 12,55 & 0,6 \\
\hline & & IR13.3 & 13 & 13,6 & 0,6 \\
\hline-- & MWIR & MWIR & 46 & 6.25 & 1.65 \\
\hline
\end{tabular}

Table 1 : Spectral bands of interest for FCI and IRS instruments and associated Detector Assemblies. On FCI we have 5 Detector Assemblies grouping 16 bands from visible range to long wave infrared. On IRS we have 2 large IR bands in MWIR and LWIR ranges and a dedicated Detector Assembly for each retina.

\section{FOCAL PLAN ARRAY OF FCI AND IRS DETECTORS}

\subsection{Retina designs}

Figure 1 and Figure 2 present respectively a schematic of the pixel and retina design for FCI and IRS.

The objective for FCI is to have a Linear sampling of 1 column by 112, 224 or 448 pixels (depending on the spectral channel and its specific SSD) as we will have along track continuous scanning of Earth surface. The implementation of different columns offers the possibility to select the best pixel per row and to guaranty a $100 \%$ pixel operability in the field of view. 




Figure 1. Schematic view of the specific FCI retina pixel design, implemented for each FCI sensitive channel and manufactured by Teledyne-E2V for VIS detector assembly and by LYNRED for NIR, IR1, IR2 and IR3 detector assemblies. The rhombus shape of pixels is specific for FCI so as to optimize the FTM (TAS design). Only one pixel per row is selected so as to constitute, at the end, a linear sampling along track of 112224 or 448 SSD.

On IRS (Figure 4), the two ranges MWIR and LWIR present the same pixel design on geometrical optical point of view. Their pitch is of $90 \mu \mathrm{m}$ which draws a SSD of $4 \mathrm{~km}$ on ground at the Earth equator. The field of view is a square of $160 \times 160$ pixels. Each pixels is actually the $3 \times 3$ electrical binning of smaller subpixels. In reality, the matrix is a 480x480 subpixels of $30 \mu \mathrm{m}$ pitch. This architecture, by the way of a best subpixels selection mapping, permits to get a nearly 100 $\%$ of operability in binning mode even with a challenging cut off LWIR matrix of $14.5 \mu \mathrm{m}$. At such cut-off, the photosensitive material presents inevitably localized defects at the subpixel scale which can be erased by the combination of mapping deselection and electrical binning association. The retina picture can be either of $160^{2}$ or $480^{2}$ pixels according to the serial data word sent by telecommand to the detector. The constrains of interferograms imposed to get a $160^{2}$ pixel picture at the frame cadency of approximately $2500 \mathrm{~Hz}$. With a pixel frequency of $4 \mathrm{Mhz}$, the frame frequency is possible if the retina is read with 16 parallel video chains (each one of $160 \times 10=1600$ pixels). The retina read out circuit is versatile that is to say it has been designed so as to be compatible for MWIR as well as LWIR photosensitive material. The corresponding pixel capacitance size can be selected according to the photosensitive material hybridized above them. The Read Out Circuits are classical with a reception stage of biases and master clock, and, by the way of multiplexing stages and video amplifiers, they deliver an analog video Dynamic Range signal of 2-3V towards the remote video electronic boards (VCU).

During the acquisition of successive interferogram frames before Fourier transform spectral reconstruction, the line of sight of the pointed field of view is maintained static. At the end of each interferogram, the line of sight is shifted by a step of approximately 158 pixels (the difference of 2 pixels wrt the 160 pixels of a complete field of view assures an overlay of 2 pixels between neighbor fields of view). Again, interferograms are acquired in this static field of view position. The operation is repeated step by step to cover at the end the entire Earth hemisphere seen at geostationary orbit. 


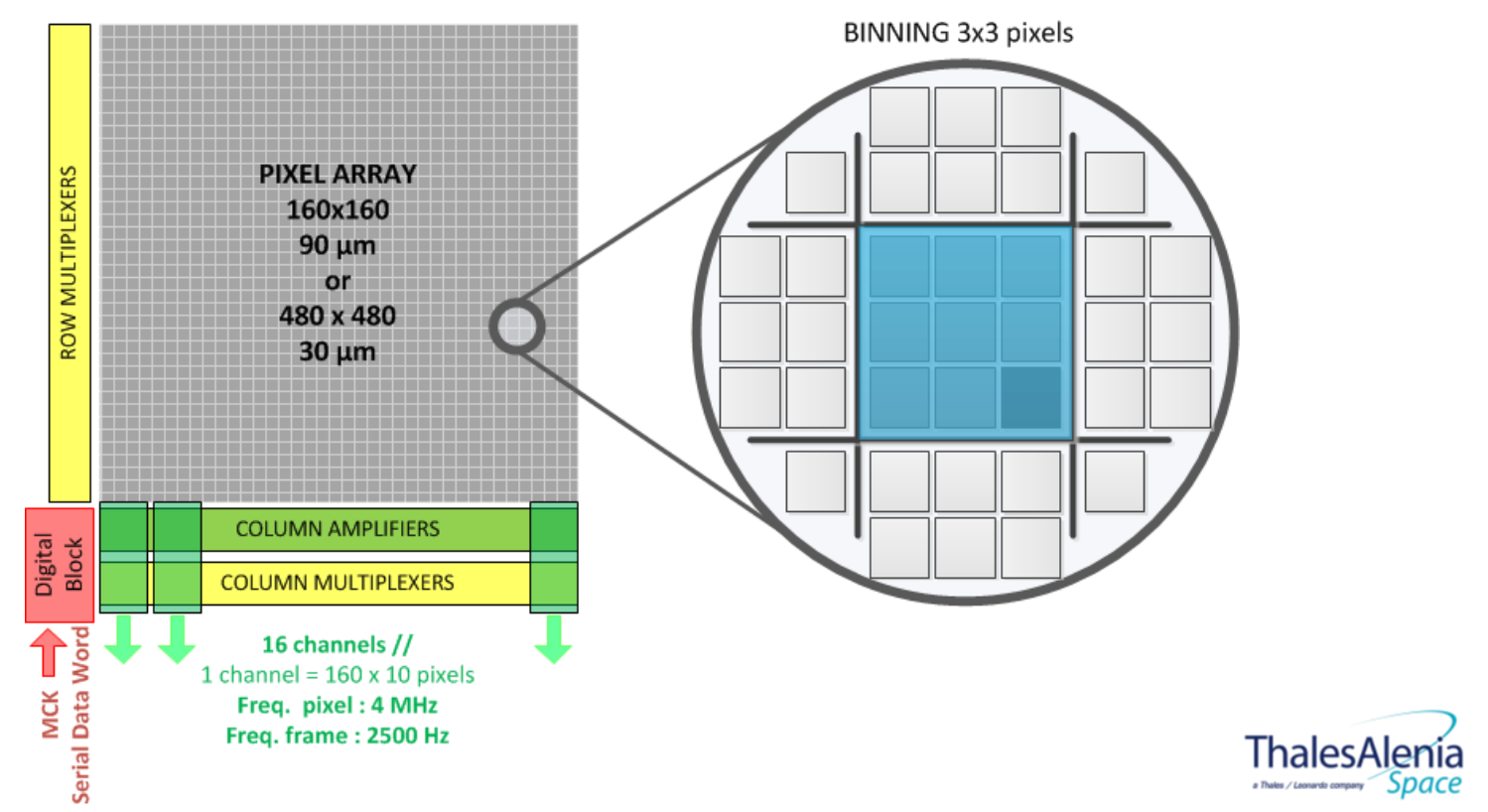

Figure 2. IRS retina with its squared pixels inscribed in a squared field of view of 160x160 pixel. Each pixel is composed of a binning of $3 \times 3$ subpixels. The ROIC (hybridized underneath the HgCdTe photosensitive material) is the same for each band (MWIR and LWIR). The capacitance integration size can be selected wrt the spectral band (MWIR or LWIR)

Commonly, the FCI and IRS detectors are based on a snapshot picture acquisition combined with an Integrated While Read protocol based on CMOS pixel addressing and read out. Integration times can be programmed until the maximum frame period duration which is fixed and common for all detectors of an instrument. The photodetectors are in Silicon for visible range and in $\mathrm{HgCdTe}$ for NIR and IR domain.

A final common constrain for FCI and IRS is to guaranty a perfect superposition of the field of view between the different spectral field of view seen by each detector in order to make an accurate spatial superposition of the spectral information. Then each detector can be adjusted in focus but also in lateral position in the focal plan array to superpose the pictures. This operation is made and controlled during the on ground integrations and tests.

\subsection{Dispositions of detectors in the focal plans}

The common entrance beam is split into five different spectral beams for FCI and two beams for IRS by the way of dichroic beam splitters.

On FCI, the most critical disposition is for the four IR detectors which are necessitating to be cooled at cryogenic temperature of $60 \mathrm{~K}$. The detectors are fixed on a cooled table and put side by side together to form a square as depicted by Figure 3

On IRS, the situation is the same but with only two detectors. The detectors are put in front each side of a dichroic beam splitter and they are fixed on a cryogenic "cold box" inserted in a cryostat (Figure 4). The working temperature is of 55 $\mathrm{K}$.

Cryogenic temperature is necessary to limit the shot noise of the dark current and to guaranty the SNR objective of all IR spectral channels. 


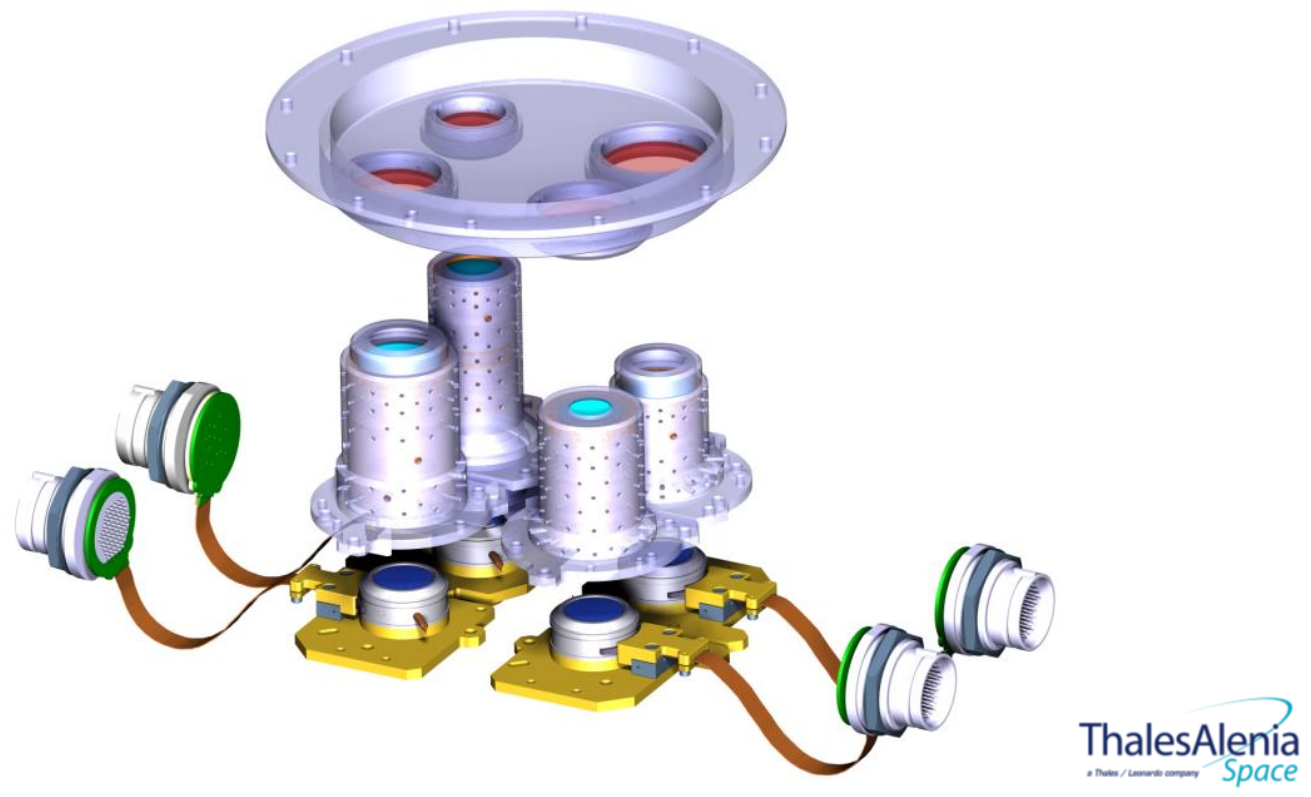

Figure 3 : A view inside the FCI cold cryostat, from the top to bottom we have successively the cryostat windows, the cold optics, the detector assemblies (window and retinas with ROIC), the detectors' baseplates (yellow) fixed on the cold table $(<60 \mathrm{~K})$. Flexible $\&$ connectors on each side are making the link to the front end electronics and the ADC for detector commands and video signal acquisitions.

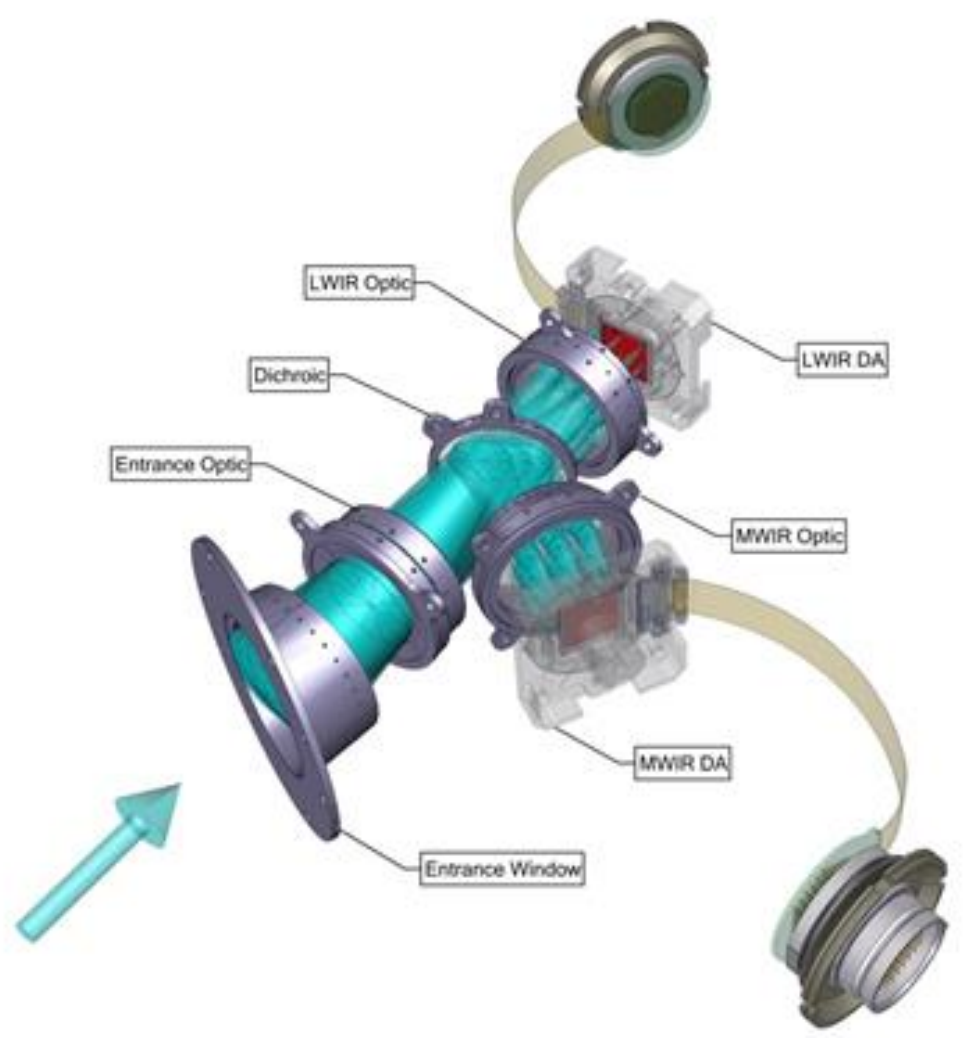

Figure 4 : MWIR and LWIR detectors are fixed at each side of a dichroic beamsplitter dividing the entrance beam in MWIR and LWIR beams. 


\section{THE DETECTION CHAINS : DESIGNS AND HARDWARES}

\subsection{The Detector Assemblies}

The five FCI Detector Assemblies (Figure 5 \& Figure 6) are constituted of a retina (formed of the photosensitive material and the Read Out Integrated Circuit), a filter and a protective window above the retina, a flexible cable makes the electrical link between on one side the retina and the video electronics by a connector brazed at the other side. A black shield is surrounding the retina to limit the optical straylight. All is fixed on a base plate, at instrument temperature for visible detector, at $60 \mathrm{~K}$ for IR detectors.

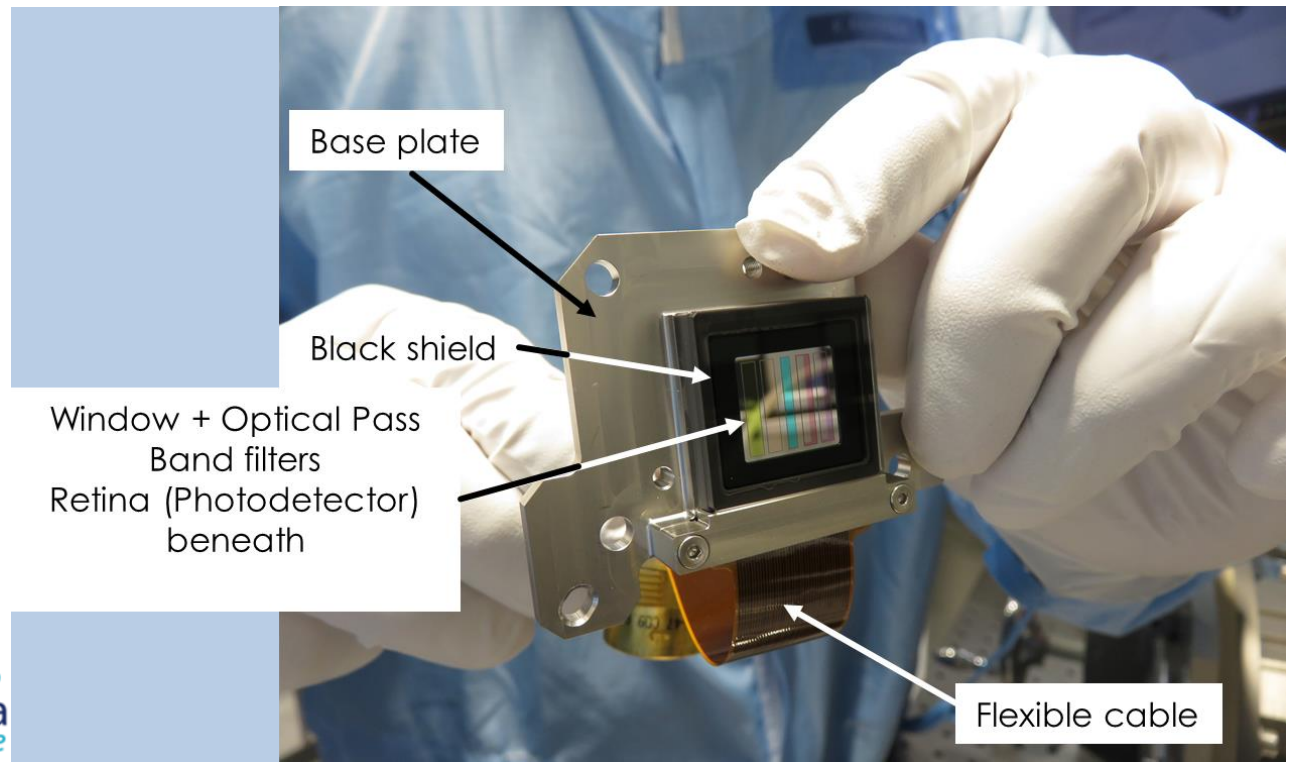

Figure 5. FCI visible detector assembly manufactured by Teledyne-E2V

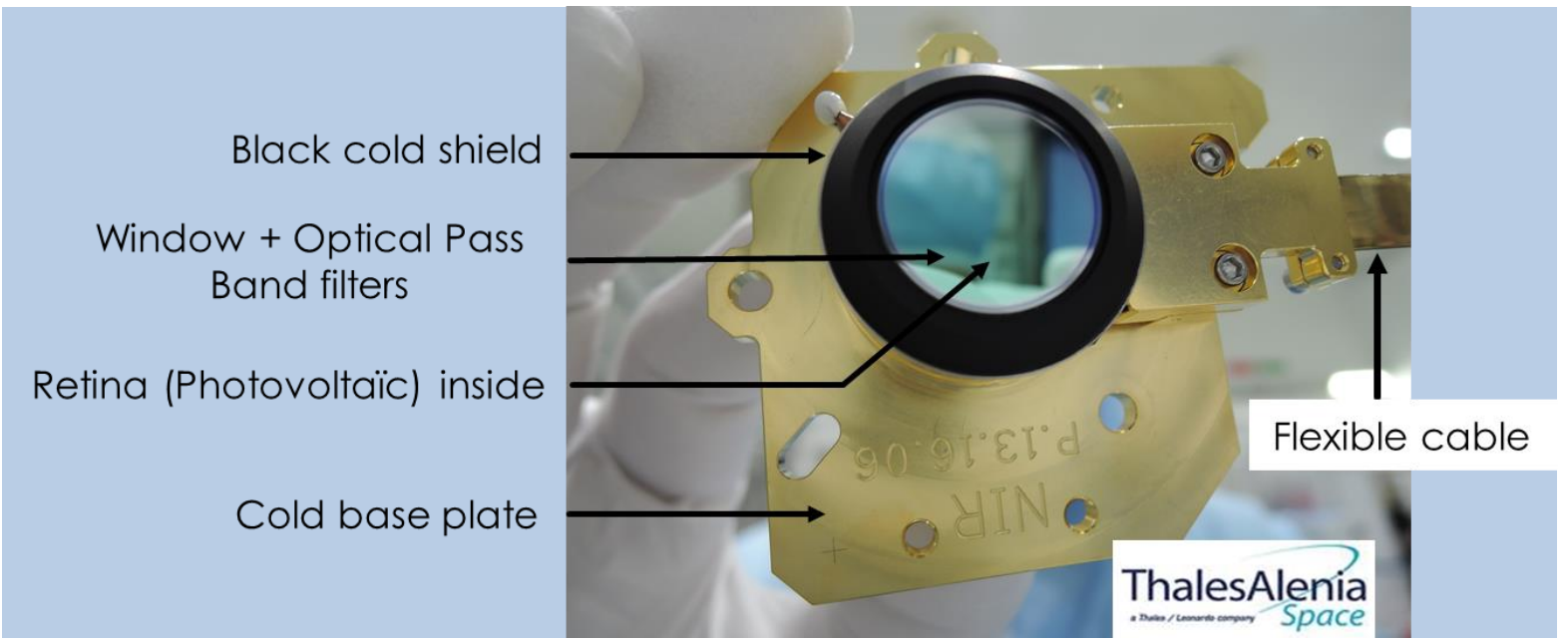

Figure 6. FCI IR detector assemblies manufactured by LYNRED : here is the picture of a NIR detector. Under the protective window, NIR Retinas 1.3, 1.6 and 2.2 are fixed with their respective optical band pass filters. The electrical link is made by the way of a flexible cable on the right of the picture. The flexible cable is joining the ROIC of the retina to the front end electronic via a connector. The base plate is cooled at $60 \mathrm{~K}$.

The two IRS Detector Assemblies (Figure 7) are composed of a retina, of a flexible cable that makes the electrical link between the retina and the video electronics by a connector brazed at one side. A black shield is surrounding the retina to limit the optical straylight. All is fixed on a $55 \mathrm{~K}$ base plate. Contrary to FCI, no windows nor filter are present. The 
optical band pass selection is made by an association of instrument cut-on optics and photosensitive retina material cutoff wavelength.

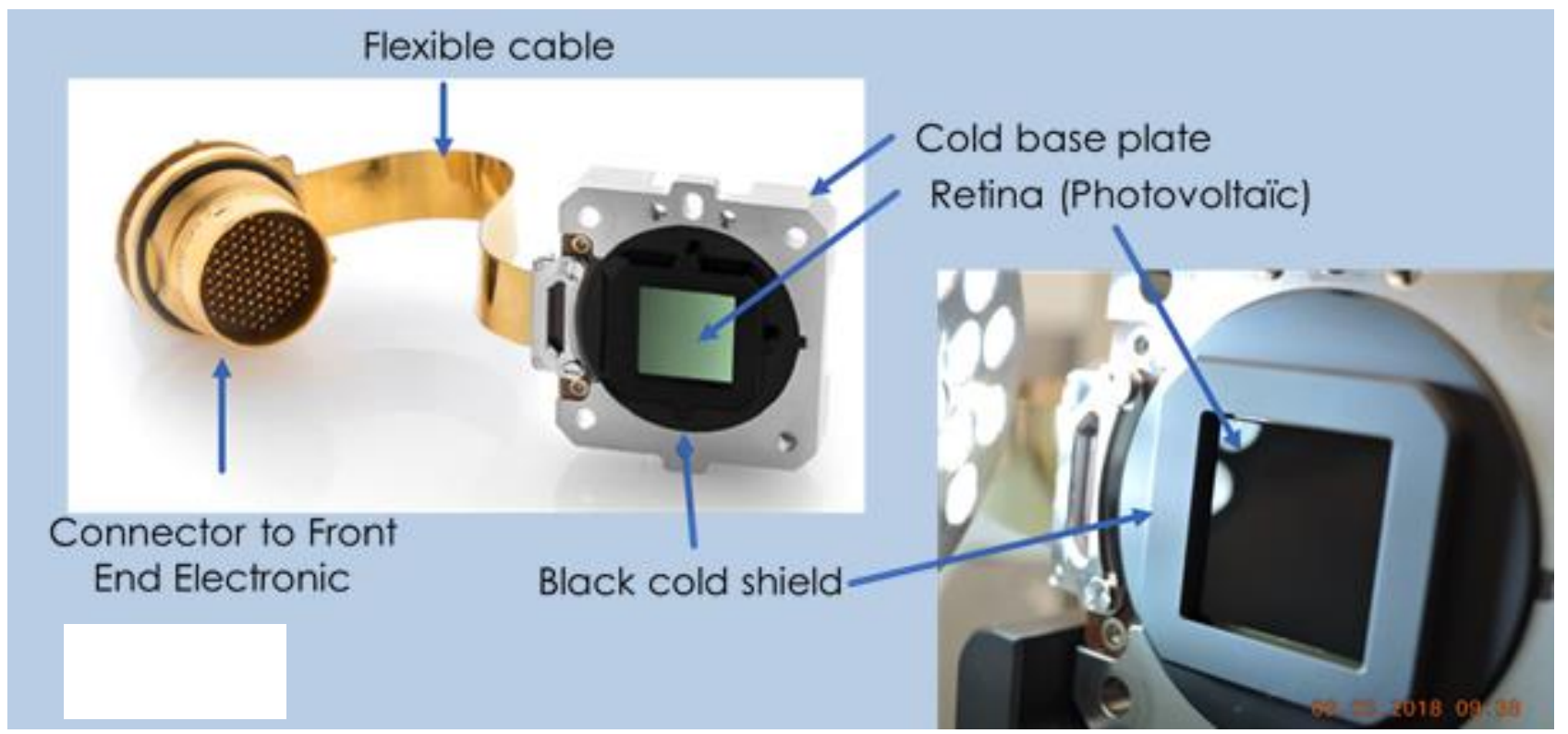

Figure 7. The IRS Detector Assembly. The retina is fixed on a molybdenium 55K base plate, surrounded by a dark cold shield. No protective windows are installed above the retinas. On the left, The electrical linked is made by the way of a flexible cable. The flexible cable is joining the ROIC of the retina to the front end electronic via a connector (left extremity in the picture).

\subsection{The video Chain electronics}

The Video Chain Electronics (VCU) are on both instrument FCI and IRS constituted of the same elements with the same principle of architecture. Hence, we describe the IRS detection chain architecture as it is supposed to be the most representative one because of the number of pixels, the high counts of digitalized video signals and its remote functions (for the detectors and for the interferometer).

The VCU is acquiring the video information from the detectors, adapting it to the ADC stage (VAE) and transmitting it to the remote Data Processing Unit (DPU) through a single high speed serial link. Also, the VCU is managing the DA functionalities and making a synchronization with the interferometer metrology data.

As shown in Figure 8, the VCU-S is composed of the following elements:

- a Front-End Electronic modules (FEE), one per detector (LWIR and MWIR), each one conditioning 16 video channels, providing supply, bias voltages and clocks to the detectors and also including the detector temperature measurement interfaces.

- One Video Acquisition Electronic module (VAE) able to process 32 video channels (16// video chains per detector), record metrology signals (coming from the interferometer) and provide all these scientific data to Data Processing Unit through a High Speed Serial Link.

- Harness between FEEs and VAE.

- Harness between Detector Assemblies and FEEs.

- Harness between VAE and DPU

The architecture has been designed with objective of the optimum reliability, maximum video performance (lowest noise) and to offer easy and fine adjustments of the analog DA signal to benefit of the maximum available conversion 
swing. Power supply distribution of FEE and DA has been defined at VCU level to reach the very low noise requirements at FEE/DA interface. During PFM campaigns, the VCU noise contribution has been measured lower than 1.6 LSB16 on a dynamic of $65536\left(2^{16}\right)$ levels ( Figure 9)
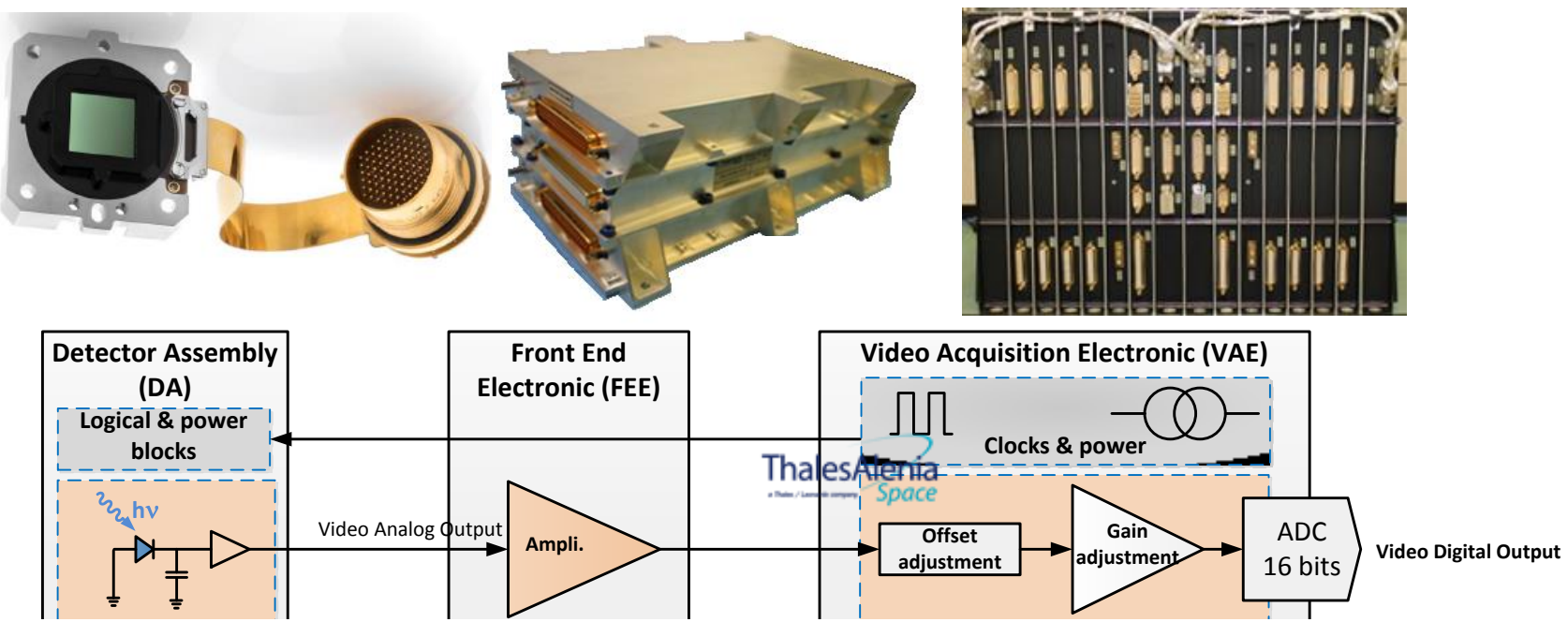

Figure 8. The full video electronic chain from the DA signal acquisition to the digitalized video signal. The video control electronics unit linked to the detectors reconstructs the full picture and provide it for processing unit (DPU) at the necessary $2500 \mathrm{~Hz}$ frequency by the way of a high speed serial link design (HSSL).

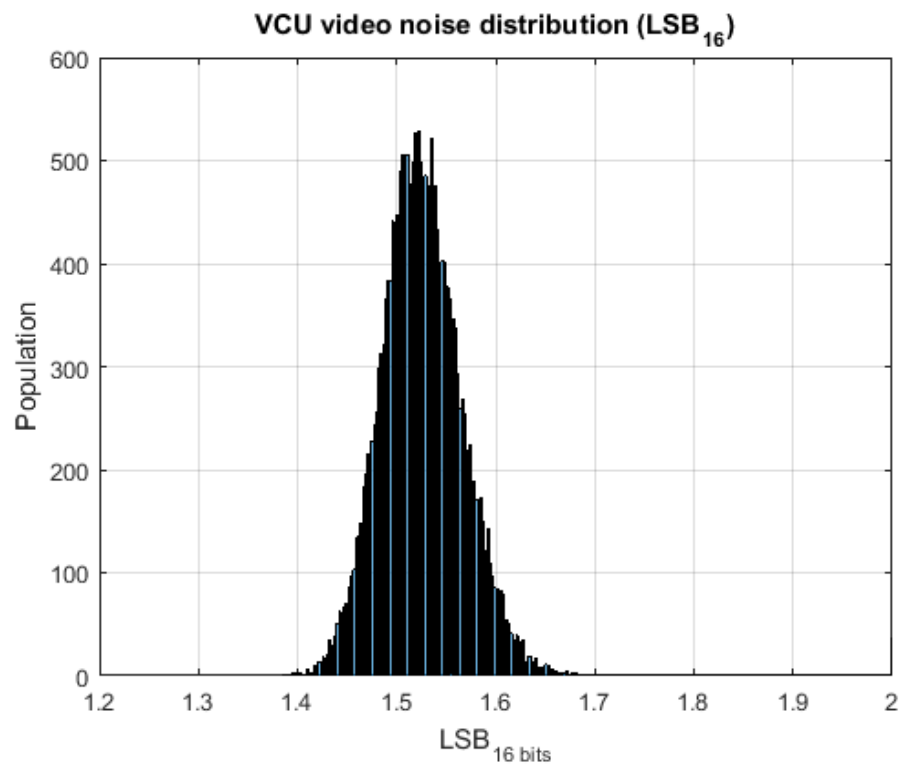

Figure 9 : VCU-S video noise measured without detector (blanked acquisition). The VCU video noise contribution is typically around 1.5 count on 16 bits and with a very low dispersion between video channels and pixels. Similar performance obtained on FCI video chain unit.

\section{SETTING AND REMOTE FUNCTIONS OF THE DETECTION CHAINS}

By the way of the Video Chain Unit and DA design, few functions are available to select different modes of DA acquisitions but also to optimize some settings and to benefit of smart remote processing. 
These affordable functions on the detection chains are summarized hereafter :

- the integration time can be adjusted from nearly 0 until the maximum time of a frame period duration. This is permitting to adapt and to maximize the integration time to the real conditions of the input photonic flux on different spectral range so as to maximize the SNR.

- An offset level and the Video Electronic amplification gain are adjustable to fit the real DA video signal Dynamic Range (from minimum to maximize illumination at the programmed integration time) with the ADC swing.

- The sampling instant of video analog to digital conversion can be positioned in the most stable window of the pixel period sequencing.

- The DA pixel selection mapping can be injected in the DA remote memory in order to have a $100 \%$ pixel operability in the pictures. A control of the written memory is available to prevent on bad programming or SEU-SEE disturbances in the DA memory.

- The picture resolution on IRS detectors can be switched from $160^{2}$ pixels (pixel binning $3 \times 3$ ) to the finest spatial resolution of $480^{2}$ pixels.

- A pseudo Correlated Double Sampling function is available to reduce the low frequency frame to frame noise if any.

- A non-linearity correction of the video signal can be performed by sending appropriate coefficient corrections of each pixels.

- Dark current skimming function on IRS avoids the dark level contribution in the useful video signal swing of conversion so as to benefit of the plenty ADC swing conversion, to afford maximum contrasted digitalized pictures

- Antiblooming functions are implemented in pixels to avoid or strongly limiting any disagreement at high flux (depolarization, smearing).

- Latch up protection with current limitation to protect the detection chain of any current burst (in orbit irradiation flaring, accidental in field of view Sun intrusion). 


\section{PERFORMANCE}

Significant performance are indicated for FCI on Table 2 and for IRS on Table 3.

\begin{tabular}{|c|c|c|c|c|}
\hline DA & Channel & $\begin{array}{l}\text { SNR (minimum } \\
\text { ill.level) }\end{array}$ & $\begin{array}{c}\text { NEDT [K] (ref ill. } \\
\text { level) }\end{array}$ & $\begin{array}{c}\text { spectral bandwidth } \\
{[\mu \mathrm{m}]}\end{array}$ \\
\hline \multirow{5}{*}{ VIS } & VIS0.4 & 26 & - & 0,06 \\
\hline & VIS0.5 & 26 & - & 0,04 \\
\hline & VIS0.6 & 22 & - & 0,05 \\
\hline & VIS0.8 & 29 & - & 0,05 \\
\hline & VIS0.9 & 28 & - & 0,02 \\
\hline \multirow{3}{*}{ NIR } & NIR1.3 & 52 & - & 0,03 \\
\hline & NIR1.6 & 51 & - & 0,05 \\
\hline & NIR2.2 & 7 & - & 0,05 \\
\hline \multirow{3}{*}{ IR1 } & IR3.8 & - & 0.13 & 0,4 \\
\hline & IR6.3 & - & 0.03 & 1 \\
\hline & IR7.3 & - & 0.04 & 0,5 \\
\hline \multirow{2}{*}{ IR2 } & IR8.7 & - & 0.03 & 0,4 \\
\hline & IR9.7 & - & 0.04 & 0,3 \\
\hline \multirow{3}{*}{ IR3 } & IR10.5 & - & 0.13 & 0,7 \\
\hline & IR12.3 & - & 0.08 & 0,6 \\
\hline & IR13.3 & - & 0.12 & 0,6 \\
\hline
\end{tabular}

Table 2. Performance SNR NEDT of FCI detection chain

\begin{tabular}{|l|l|l|}
\hline IRS & MWIR & LWIR \\
\hline Band & $4.6 \mu \mathrm{m}-6.25 \mu \mathrm{m}$ & $8.26 \mu \mathrm{m}-14.3 \mu \mathrm{m}$ \\
\hline SNR (typ), Scene at 270 K & $>3100$ & $>7700$ \\
\hline $\begin{array}{l}\text { Total video noise with photonic noise at reference flux } \\
\text { scene of 270 K (rms counts 16 bits) }\end{array}$ & 10 & 5 \\
\hline VCU video noise contribution (rms counts 16 bits) & 1.6 & 1.6 \\
\hline Pixel operability & $100 \%$ & $100 \%$ \\
\hline Signal non linearity (along the full signal DR) & $<0.5 \%$ & $<0.5 \%$ \\
\hline Video Gain stability on 1h & $<0.02 \%$ & $<0.02 \%$ \\
\hline PRNU (binning mode) & $<2 \%$ & $<2 \%$ \\
\hline
\end{tabular}

\section{Table 3. Main performance of IRS detection chain}

The IRS uniformity of pixel induces on both spectral ranges a low PRNU and a high SNR (typical above 3100 in MWIR and 7700 in LWIR) with a Gaussian distribution for an input uniform scene illumination at $270 \mathrm{~K}$. The map of the SNR 
and the associated histograms are presented on Figure 10. These figures have been obtained without any equalisation nor non uniformity corrections : they are video data results immediately grabbed at the output of the ADC without any special processing. The pictures highlight the field SNR performance uniformities of the entire pixel population that constitutes a key performance for a spectro-imager like IRS.
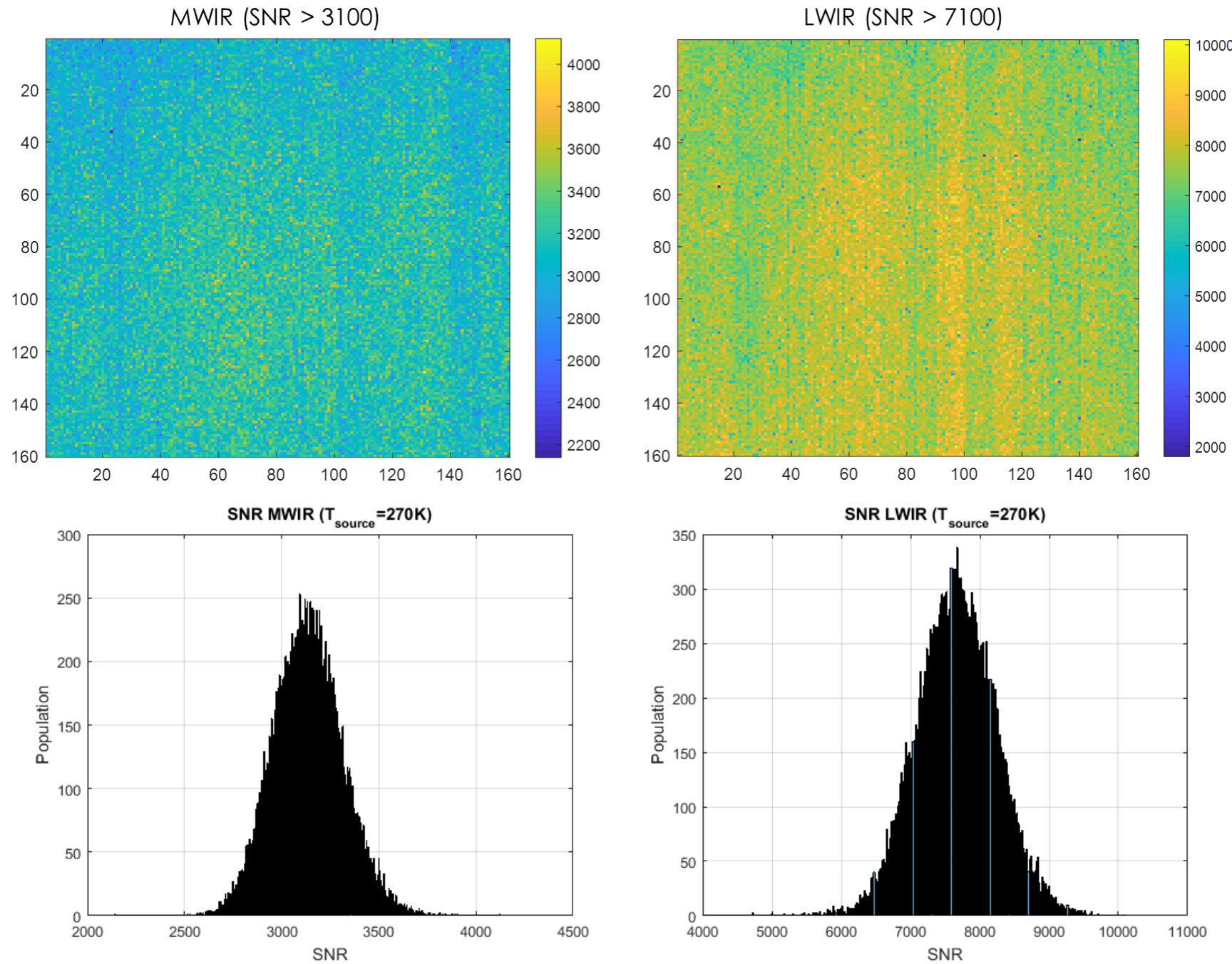

Figure 10. SNR in field of view and histogram for a black body source at reference illumination of $270 \mathrm{~K}$.

The SNR performance combined with spectral response of the instrument, after spectral interferograms reconstruction is expected to provide a spectral NEDT. This spectral NEDT indicated on Figure 11 gives the ultimate performance the IRS instrument can reach on each spectral channel. 

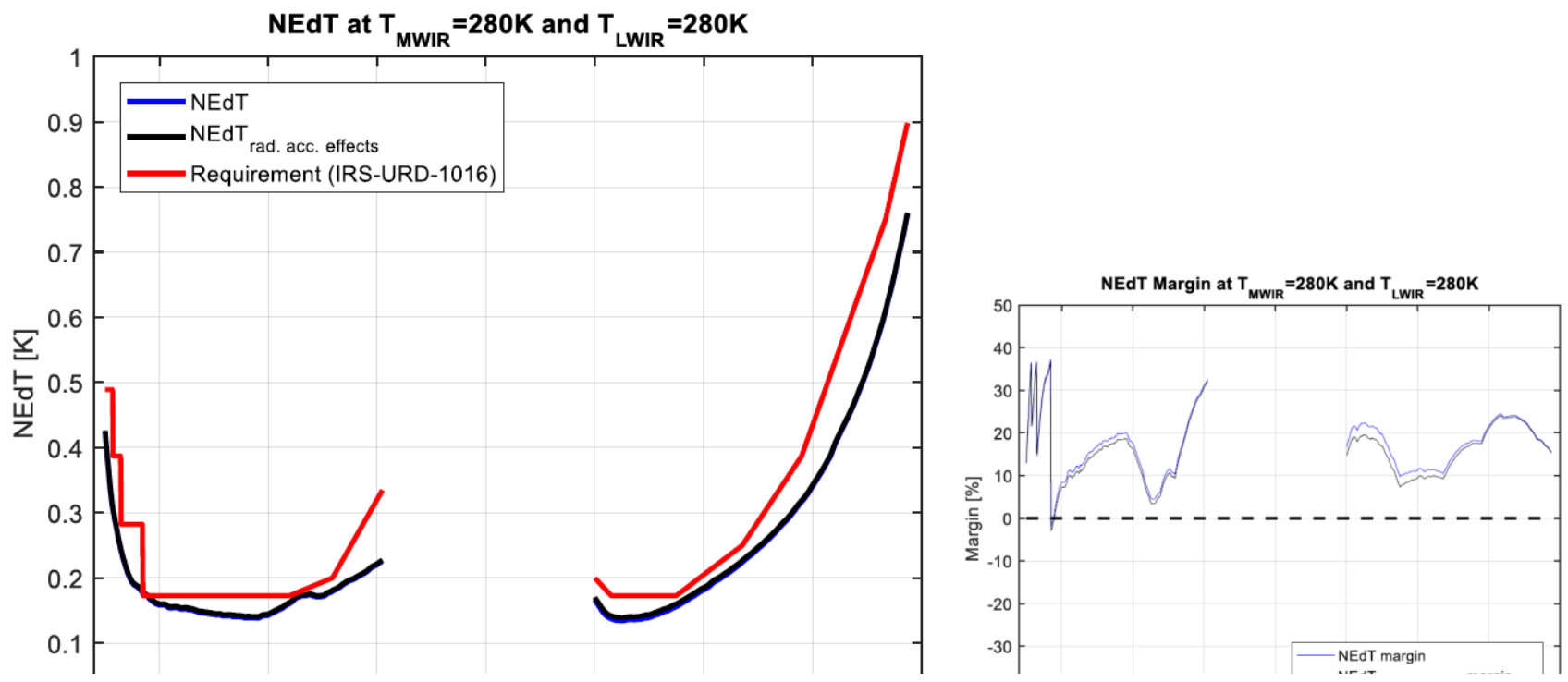

Figure 11. IRS NedT estimation for LWIR and MWIR, based on typical detector PFM performance and full instrument IRS characteristics, at an illumination of reference equivalent to a black body of $280 \mathrm{~K}$. On the left, the NEDT performance takes into account the full instrument optical transmissions, the straylight and out of band parasitic signals, the total temporal noise of the detection chain and its spectral response as well as the data processing noises to reconstruct the spectrum of the observed irradiance. On the right the corresponding margins with regards to the objectives of performance (requirements). Source credit OHB.

\section{CONCLUSION}

FCI and IRS PFM detection chain fully designed and integrated by Thales Alenia Space have been characterized on PFM.

Major performance of spectral SNR, NEDT, linearity and pixel operability are in line with expectations and objectives.

Sub equipment (Detectors, Video Chain Electronics) have been successfully tested to environments (in particular for vibrations, shock, life test and irradiations)

IRS PFM will be delivered by Thales Alenia Space to OHB Munich beginning of this year for a complete and final IRS instrument integration, to check full functionalities and performance characterization of the complete device.

MTG FCI \& IRS FM2 are today in progress of integration in Cannes premises.

Thales Alenia Space is fully grateful to OHB for close partnership on MTG, to ESA for technical and management supervision, to ESA and EUMETSAT for supports and confidence in Meteosat program and to all the subcontractors who have designed and provided the best sub-equipment for the tomorrow meteorological forecasts. Concerning the detection chain subequipment, we specially acknowledge LYNRED, E2V TELEDYNE, Thales Alenia Space in Spain.

\section{REFERENCES}

[1] Martin, P \& Al, "FCI instrument on-board MeteoSat Third Generation Satellite - design and development status", ICSO 2020, (2021)

[2] Ouaknine, J. Gaudin-Delrieu, C. Liotard, A, Boni, A. , Aminou, D. Durand, Y., "FCI PFM optical test results", ICSO 2020, (2021) 
[3] Siméoni, D \& Al, "Design and development of IASI instrument', Proc. SPIE. 5543, Infrared Spaceborne Remote Sensing XII, (04 Nov. 2004)

[4] Blumstein, D \& Al, "IASI instrument: technical overview and measured performances", Proc. SPIE. 5543, Infrared Spaceborne Remote Sensing XII, (04 Nov. 2004)

[5] European Space Agency, "Meteosat Third Generation (MTG) Space Craft System Requirements Document (SC_SRD) [AD03]”, ref. MTG.ESA.SA.RS.0062 issue 3.3 (23 October 2013)

[6] European Space Agency, "Meteosat Third Generation (MTG) Terms, Definitions, Conventions and Abbreviations [AD16]", ref. MTG.ESA.SA.RS.0052 issue 4.1 (02 September 2013) 